\title{
Design and Development of Coded Aperture Compressive Sensing Acquisition for High Frame Rate TEM Imaging
}

\author{
L. Kovarik ${ }^{1}$, A. Stevens ${ }^{2,3}$, A. Liyu ${ }^{1}$, J. Davidson ${ }^{4}$, R. Billhorn ${ }^{4}$, N.D. Browning ${ }^{5,6}$ \\ ${ }^{1 .}$ Environmental Molecular Sciences Laboratory, PNNL, Richland, WA \\ 2. National Security Directorate, PNNL, Richland, WA, USA \\ 3. Duke University, Electrical and Computer Engineering, Durham, NC \\ 4. Direct Electron, LP, San Diego, CA, USA \\ 5. Physical and Computational Science, PNNL, Richland, WA, USA \\ ${ }^{6 .}$ Materials Science and Engineering, University of Washington, Seattle, Washington, USA
}

Transmission Electron Microscopy (TEM) enables to probe the dynamics of materials with spatial and temporal resolution that can vary over several orders of magnitude. In several research fields, it is critical to capture the materials behavior at high temporal resolution but due to a limited detector readout rates, the temporal resolution is currently limited mostly to milliseconds. To overcome these limitations, novel concepts of recording high frame video in TEM have been recently explored. Among those concepts, tiling of images on a detector using fast electrostatic deflectors has demonstrated the opportunity to acquire small video series at nanosecond resolution [1,2]. In this work we investigate an alternative approach of acquiring high frame-rate video using a coded aperture acquisition and compressive sensing (CS) reconstruction [3]. In this presentation, we will discuss the development of coded aperture imaging system and present initial results.

The coded aperture video acquisition can be understood as a process of integrating multiple spatially coded frames into a single camera frame [3,4]. The individual frames can be recovered from the integrated frame with the use of compressive sensing (CS) reconstruction algorithm. The principle of coded aperture CS video acquisition is shown in Figure.1a. The coded aperture, which is the most critical component of CS acquisition system, has to meet several unique constraints for electron optics applications. Namely, it has to maintain self-supporting nature, which imposes constrains on the modulation function, and it also has to provide modulation at the pixel level, which is associated with manufacturing difficulties. Both design considerations will be discussed in the context of our current design. The depiction of coded aperture, and its modulation function, is shown in Figure1(b,c).

In our acquisition setup, the coded aperture is controlled by piezoelectric driven stage, which was implemented in a custom designed acquisition module based on Direct Electron DE20 camera. The newly built acquisition system was tested on aberration corrected FEI Titan 80-300, and the experiments were performed with a standard $\mathrm{Au}$ grating replica sample under various degree of translation of $\mathrm{Au}$ grating sample. In the current implementation, successful reconstructions can be achieved only under limited conditions. An example of video compressive sensing acquisition under a negligible translation of the sample is shown Fig.2(b,c,d). Not coded image of Au grating sample used for CS video acquisition is shown in Fig. 2(a). As a part of this presentation we will discuss how larger translation of the $\mathrm{Au}$ grating sample presently affects the resolution of the reconstructions. Factors that limit the applicability CS video reconstruction will be discussed, together with the future outlook for implementation of this technique for high frame rate acquisition [5]. 


\section{References:}

[1] LaGrange T., Reed B.W, Masiel D. J., MRS Bull. 40 (2015), p. 22.

[2] Reed, B. W., Park, S. T., \& Masiel, D. J., Microscopy and Microanalysis 22(S3) (2016), p. 706.

[3] Llull, P. et al, Optics Express 21(9) (2013), p. 10526.

[4] Stevens, A. et al, Advanced Structural and Chemical Imaging 1(10) (2015), p. 1.

[5] This research was part of the Chemical Imaging Initiative at Pacific Northwest National Laboratory. The work was conducted in the William R. Wiley Environmental Molecular Sciences Laboratory (EMSL), a national scientific user facility sponsored by DOE's Office of Biological and Environmental Research and located at PNNL.

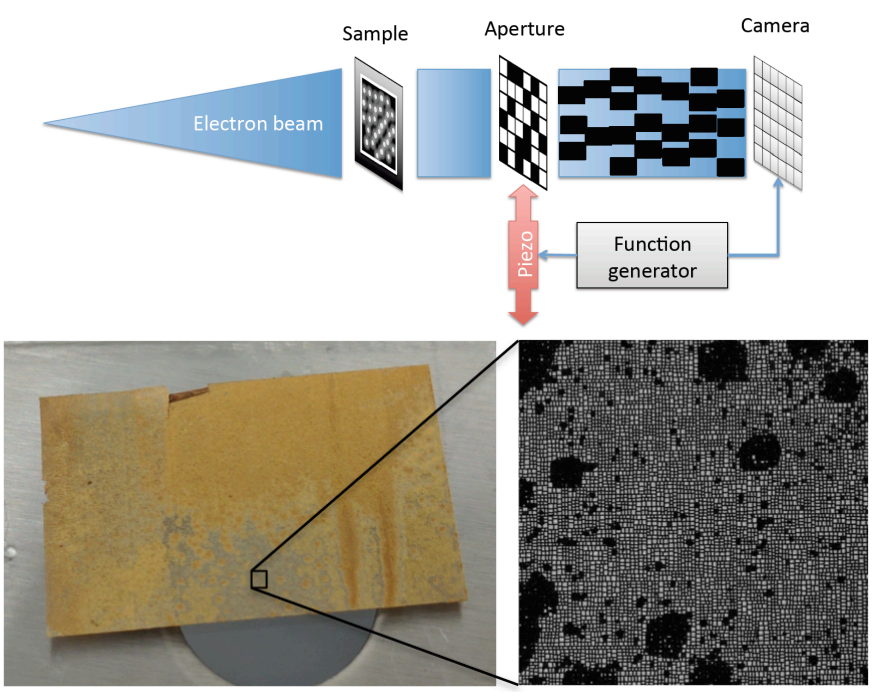

Figure 1. (a) Schematic diagram of coded aperture acquisition setup (b) The code aperture developed for video compressive sensing acquisition (c) Magnified view of aperture under white illumination. Defects in the aperture (black patches) limit the transmissivity of the aperture.
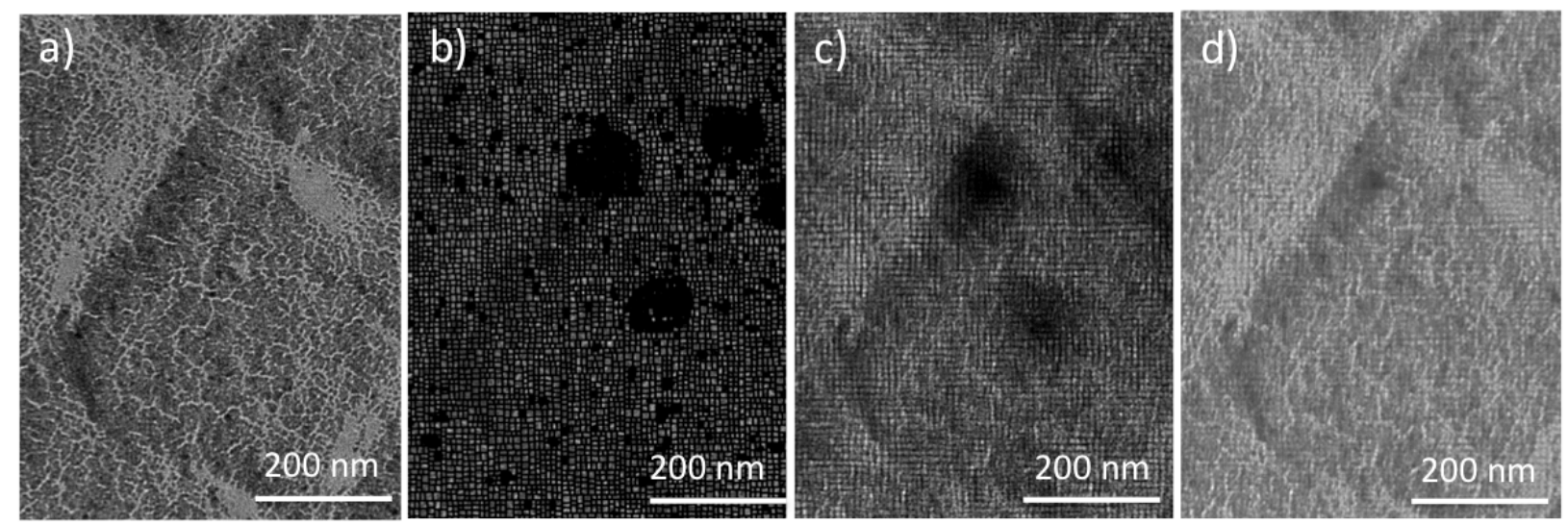

Figure 2. (a) Original (not-coded) view of Au grating sample used for CS video acquisition. (b) Individually coded frame (c) Integration of 16 coded frames (d) Individual frame recovered from the integration of 16 frames. 